Canadian
JOURNAL
MEDICINE

\title{
An Evaluation of the Potential of Heparin to Inhibit the Viral Entry of SARS-CoV-2
}

\author{
Sudipta Samadder, ${ }^{1,}$, Peter Anto Johnson ${ }^{1,3 *}$, John Christy Johnson ${ }^{1,3}$, Austin \\ Mardon $^{1,3}$
}

${ }^{1}$ Antarctic Institute of Canada, Canada

${ }^{2}$ Department of Biochemistry and Biomedical Sciences, McMaster University, Hamilton ON, Canada

${ }^{3}$ Faculty of Medicine and Dentistry, University of Alberta, Edmonton AB, Canada

\section{Keywords: \\ Heparin, SARS-CoV-2. \\ Polysaccharide, COVID-19, \\ Glycosaminoglycans, \\ Inhibition}

\section{Received}

18 June 2021

Received in revised form

29 July 2021

Accepted

01 August 2021

*Correspondence:

paj1@ualberta.ca

\begin{abstract}
Heparin is an anticoagulant medicine that prevents the formation of harmful blood clots in the vessels. Following the outbreak of the novel coronavirus disease 2019 (COVID-19), heparin has helped to improve the health of affected patients beyond its anticoagulant effects. The potential antiviral activity of heparin has attracted speculation due to its highly sulfated profile, which allows it to have a high binding affinity to a wide range of viral components. Heparin's successful binding to the ZIKA virus, human immunodeficiency virus, as well as the SARS CoV and MERS CoV spike proteins have demonstrated its potential to inhibit the entry of SARS-CoV-2 into the body. A high degree of sequence homology also enables heparin to have inhibitory binding potential on viral components. The SARS-CoV-2 virus exhibits significant differences in its spike glycoprotein (SGP) sequence compared to other coronaviruses. The SGP sequence in SARS-CoV-2 contains additional potential glycosaminoglycan (GAG) binding domains that may drive differences in the attachment and entry process of the virus. Findings from unbiased computational ligand docking simulations, pseudotyped spike protein experiments, and cell to cell fusion assays have also opened possibilities to investigate the antiviral properties of heparin in clinical trials.
\end{abstract}

CCIKD Publishing

Heparin is a well-known anticoagulant agent that belongs to the family of polyanionic polysaccharides called glycosaminoglycans (GAGs). Members of this family consist of alternating uronic acid and amino acid residues linked by glycosidic bonds [1]. Heparin is made 
of several hexuronic acid and d-glucosamine residues, giving it the appearance of a long, linear sulfated chain with the highest negative charge density of any known biomolecule [2]. The strong anticoagulant property of heparin can be traced to its eight- or more-repeating disaccharide units that contain the pentasaccharide sequence. This sequence creates binding sites for specific clotting proteins [3]. Heparin's most well-characterized protein interaction is with the serine protease inhibitor called antithrombin-III (AT3), which gives heparin the ability to catalyze the inactivation of factor Xa or thrombin [2]. This interaction simultaneously inhibits both thrombin generation and thrombin activity in the blood and consequently inhibits blood coagulation. Despite high incidences of coagulation in the early stages of infection from the novel coronavirus disease 2019 (COVID-19), anticoagulant therapy has been found to provide the greatest protection against the disease progressing to its hyperinflammatory stage [4]. Many other pharmacological properties of heparin have been identified, including anti-inflammatory, anti-viral, anti-angiogenesis, anti-neoplastic, and antimetastatic properties [3]. In spite of mounting research on heparin's effectiveness in treating the adverse side effects of COVID-19, there is still limited clinical data linking heparin therapy to meaningful antiviral outcomes. In this review, Heparin is evaluated as a potential antiviral agent to inhibit the entry of the SARSCoV-2 virus and improve treatment for COVID-19.

\section{Antiviral Effect of Heparin in Preclinical Studies}

Heparan sulfate proteoglycans (HSPGs) are cell membrane glycoproteins consisting of proteins covalently coupled with heparin sulfate (HS) chains [5]. HS is a linear polysaccharide composed of repeated units of disaccharides. The extracellular polysaccharides bind to a wide range of ligands and enable their use as a cell surface docking site or receptor for the ligands attached to them. There has been ample evidence demonstrating that viruses and pathologic amyloid proteins bind to HS and hijack the HSPGs-mediated endocytosis pathway to internalize into host cells. Examples of viruses that use HSPG to infect cells are cytomegalovirus, respiratory syncytial virus, human papillomavirus, hepatitis-C virus, hepatitis-B virus, Zika virus, and coronavirus [5]. Research into the successful inhibitory binding of heparin to the Zika virus in particular shows promise for the antiviral activity of heparin on the novel coronavirus.

The Zika virus (ZIKV) is a member of the Flavirvirdea family [6]. In February 2016, ZIKV was declared a public health emergency of international concern by the World Health Organization [7]. The complexity of the virus is due to its elegant attachment and entry into target cells with the help of co-receptors. For a successful entry and replication inside a host cell, a virus requires a high degree of conformation between its binding site and its co-receptor [8]. In a real-time interaction study, Kim and colleagues examined the binding of the ZIKV envelope (ZIKV E) protein to GAGs to investigate the role of GAGs in the entry of ZIKV into the placenta and the brain [9]. The results from surface plasmon resonance microscopy demonstrated that the ZIKV E strongly binds $\left(\mathrm{K}_{\mathrm{D}}=443 \mathrm{nM}\right)$ pharmaceutical heparin, a highly sulfated GAG, and weakly binds to less sulfated GAGs. Various GAGs were then tested for their ability to inhibit ZIKV E and heparin interaction. Heparin showed a strong inhibition level of $95.2 \%( \pm 0.3)$ in comparison to other GAG structures, including chondroitin sulfate type $\mathrm{E}$ (CSE;79.2\%, \pm 0.9 ), chondroitin sulfate type B (CSB; 71.6\%, \pm 1.2$)$, heparin sulfate (HS; $57.1 \%$, $\pm 1.6)$, Keratin sulfate $(\mathrm{KS} ; 41.4 \%, \pm 2.1)$, chondroitin sulfate type D (CSD;65.2\%, \pm 3.0 ), 
chondroitin sulfate type A (CSA;19.1\%, \pm 8.3$)$, and chondroitin sulfate type C (CSC;8.2\%, \pm 0.4 ) [9]. A closer look at the sequence alignment explains heparin's strong binding affinity to ZIKV E. The structure of heparin was found to be highly homologous to the GAG binding site of ZIKV E. The results from this study suggested that highly sulfated GAGs may be utilized as attachment factors for host cell entry of viruses due to their strong binding affinity. Similar decoy strategies have already been successful in curbing other viruses, including influenza A and dengue.

Preclinical studies on the human immunodeficiency virus (HIV) infection in vitro also demonstrated favorable antiviral effects of heparin therapy in inhibiting SARS-CoV-2 entry. In a study conducted by Ito et al., heparin was observed to have a 50\% reduction in HIV-induced pathological changes to MT-4 cells at a concentration of $7.5 \mathrm{ug} / \mathrm{mL}$ [10]. A follow-up from this study demonstrated that heparin cannot neutralize HIV directly but does hinder HIV from adhering to MT-4 cells. Furthermore, all fragmented heparins inhibited the replication of HIV in MT-4 cells significantly less than the original non-fragmented heparin, indicating reduced antiviral activity in the smaller fragments [10]. These findings suggest that heparin may be useful in blocking HIV replication in vivo, and could suggest similar outcomes for other viruses.

\section{Binding affinity of SARS-CoV and MERS-CoV Spike Glycoprotein (SGP)}

Extensive research on the SARS-CoV and MERS-CoV viruses has provided the most relevant evidence of heparin's antiviral activity against SARS-CoV-2. Several receptors, including heparan sulfate proteoglycan (HSPG), ACE2, and dipeptidyl peptidase 4 (DPP4), are involved in the fusion activation of the SARS-CoV and MERS-CoV viruses [11]. HSPG provides a binding site for the original SARS-CoV invasion at the initial attachment phase, allowing heparin to inhibit the cell entry of the virus. Using a surface plasmon resonance direct binding assay, the study found that both monomeric and trimeric SARS-CoV-2 spike glycoprotein (SGP) bind more tightly to immobilized heparin ( $\mathrm{KD}=40 \mathrm{pM}$ and $73 \mathrm{pM}$, respectively) than the SARS-CoV and MERS-CoV SGPs (500 nM and $1 \mathrm{nM}$, respectively) [11]. In particular, the sequence analysis of SGP of SARS-CoV-2 suggests that the virus has evolved to have additional potential GAG binding domains than the SARS-CoV virus. The high affinity of SARS-CoV-2 SGP for immobilized heparin may be attributed to the high density of surfacebound ligands that could promote polyvalent interactions [11]. Moreover, results from ligand docking studies suggest that the HS hexasaccharide fragment may span an interdomain channel that includes a site 2 , suggesting that HS saccharide sequences of longer lengths may bind more strongly.

\section{Binding Affinity of SARS-CoV-2 to Sulfated Polysaccharides}

The attachment and entry of the SARS-CoV-2 virus are mediated by the spike glycoprotein (SGP) [12]. Early evidence pointed to angiotensin-converting enzyme 2 (ACE2) as the SARSCoV-2 entry receptor based on biochemical interaction studies and crystal structure analysis. SARS-CoV-2 and SARS-CoV spike proteins share an amino acid sequence similarity of $76.5 \%$, resulting in a high degree of homology [13]. Additional analysis has revealed that SARS-CoV2 recognizes human ACE2 more readily than the original virus, increasing the virus's ability to infect other humans [13]. Therefore, it was predicted that the SARS-CoV-2 spike protein would have a very strong affinity for human ACE2. In addition to the interaction with its receptor, SGP has been found to bind glycosaminoglycans such as heparin sulfate, which is found on the 
surface of virtually all mammalian cells. A SARS-CoV-2 S1 receptor-binding domain (RBD) attachment to unfractionated heparin was first reported by Mycroft-West et al. with the use of surface plasmon resonance microscopy [14]. The report concluded that heparin caused RBD to undergo a conformational change, including helix and beta-sheet content alterations. Based on these changes, it has been demonstrated that RBD interacted with heparin in physiologically relevant solutions [14]. In this manner, the virus would adhere to the surface of the cell, increasing local concentrations of viral particles and increasing the likelihood of infection. A heparin/HS binding protein can be identified by amino acids conforming to the CardinWeintraub motifs "XBBXBX" and "XBBBXXBX". X is a hydropathic residue, and $\mathrm{B}$ is a basic residue (such as arginine and lysine), responsible for interacting with the sulfate groups present on heparin/HS [11]. A recent report by Clausen et al. showed that HS could interact with residues adjacent to the ACE2-binding site at the RBD of the S1 subunit of the SARS-CoV-2 trimeric $S$ protein [15]. The following includes the unbiased computational ligand docking predictions of putative heparin/HS-binding motifs on the S protein, i.e., 453-459 (RYLFRKS), 681-686 (PRRARS), and 810-816 (SKPSKRS). The 681-686 (PRRARS) site between S1 and S2 subunits was discovered to be a novel insertion that had been absent in the SARS and MERS $\mathrm{S}$ proteins. Molecular analysis found that the interaction between HS and SARS-CoV-2 trimeric $S$ protein facilitates the opening of S-protein conformation for ACE2 binding, thereby initiating viral infection [15].

To understand the mechanism of heparin/HS-S protein binding and virus infection, Tandon et al. pseudotyped the SARS-CoV-2 S protein on a third-generation lentiviral ( $\mathrm{pLV}$ ) vector and examined the impact of various sulfated polysaccharides on transduction efficiency in mammalian cells [12]. The study aimed to determine whether SARS-CoV-2 entry and attachment can be prevented by inhibiting HS interaction with SGP via the addition of exogenous sulfated polysaccharides on HEK293T cells. Several polysaccharides were found to be capable of inhibiting the transduction of pLV-s in a concentration-dependent fashion. Unfractionated heparin (UFH) and enoxaparin (a low molecular weight heparin drug) were also found to have similar inhibitory activity, with UFH having slightly higher activity [12]. Detailed studies of both pLV-S and SGP-sulfated polysaccharide interactions are needed to identify the optimal binding structure(s).

In a separate study, Tiwari et al. used a model of a cellular cell-to-cell fusion assay to show that the SARS-CoV-2 S protein-mediated cell-to-cell fusion could arise even in the absence of the ACE2 receptor [16]. In the model, the $S$ protein differentially recognized the 3-O sulfated HS structures produced by the two distinct isoforms, 3OST-3B and 3OST-5. The model also demonstrated that the S2 subunit was crucial for cell-to-cell fusion induced by the protein-3-O sulfated HSPG pathway [16]. Further evidence revealed that the molecular diversity of HS chains is vital in supporting entry, trafficking, and replication processes. Overall, the results from this study suggest possibilities of developing and testing small molecule agents against SARS-CoV-2 and highlight the effectiveness of HS mimetics in the treatment and prevention of SARS-CoV-2 replication in the body.

\section{Conclusion}

Studies on the interaction of heparin with a wide range of viral components suggested that heparin and heparin/HS interacting molecules may function as anti-SARS-CoV-2 drugs to 
prevent the entry of the SARS-CoV-2 virus. Many studies have focused on the SgP-ACE-2 pathway. However, HSPGs on host cell surfaces have also been demonstrated to play important roles in the pathology of enveloped viruses, including coronaviruses, HIV, and the Zika virus. The higher degree of binding complementarity between heparin and the SARS-CoV-2 virus compared to other strains of coronaviruses also supports the effective function of the drug as an antiviral agent. The effectiveness of viral entry inhibition can be explored further by examining the activity of heparin in more clinical trials and further investigating the underlying mechanism of its inhibition. Overall, these findings could support the development of therapeutics and antiviral agents to inhibit the attachment and internalization of SARS-CoV-2, which could effectively reduce infection rates.

\section{Acknowledgments}

Not applicable.

\section{Disclosure Statement}

No potential conflict of interest was reported by the authors.

\section{Funding Acknowledgements}

Not applicable.

\section{References}

[1] Young E. The Anti-Inflammatory Effects of Heparin and Related Compounds. Throm Res. 2008;122(6):743-752.

[2] Hippensteel J, LaRiviere W, Colbert J, Langouet-Astrie C, Schmidt E. Heparin as a Therapy for COVID-19: Current Evidence and Future Possibilities. Am Journal Physiol. 2020;139(2);211-17.

[3] Hao C, Xu H, Yu L, Zhang L. Chapter One - Heparin: An essential drug for modern medicine. Prog Mol Biol Transl. 2019; 163:1-19.

[4] Antiviral Therapy. https://www.covid19treatmentguidelines.nih.gov/antiviral-therapy/ [accessed 5 April 2021].

[5] Tavassoly O, Safavi F, Tavassoly I. Heparin-binding Peptides as Novel Therapies to Stop SARS-CoV-2 Cellular Entry and Infection. Mol Pharmacol. 2020;98(5):612-19.

[6] Plourde AR, Bloch EM. A Literature Review of Zika Virus. Emerg Infect Dis. 2016;22(7):1185-92.

[7] WHO. Zika: Then, Now and Tomorrow. https://www.who.int/emergencies/zika-virus/articles/then-now-hugonnet/en/ [accessed 7 April 2021].

[8] Maginnis MS. Virus-Receptor Interactions: The Key to Cellular Invasion. J Mol Biol. 2018;430(17):2590-611.

[9] Kim SY, Zhao J, Liu X, Fraser K, Lin L, Zhang X, et al. Interaction of Zika Virus Envelope Protein with Glycosaminoglycans. Biochemistry. 2017;56(8), 1151-62.

[10] Ito M, Baba M, Sato A, Pauwels R, Clereq ED, Shigeta S. Inhibitory Effect of Dextran Sulfate and Heparin on the Replication of Human Immunodeficiency Virus (HIV) in vitro. Antivir Res. 1987;7(6):361-7.

[11] Kim SY, Jin W, Sood A, Montgomery DW, Grant OC, Fuster MM, et al. Characterization of Heparin and Severe Acute Respiratory Syndrome-related Coronavirus 2 (SARS-Cov-2) Spike Glycoprotein Binding Interaction. Antivir Res. 2020;181:104873

[12] Tandon R, Sharp JS, Zhang F, Pomin VH, Ashpole NM, Mitra D, et al. Effective Inhibition of SARS-CoV-2 Entry by Heparin and Enoxaparin Derivatives. J Virol. 2021;95(3):e01987-20.

[13] Zhang H, Penninger JM, Li Y, Zhing N, Slutsky AS. Angiotensin-converting enzyme 2 (ACE2) as a SARS-CoV-2 receptor: molecular mechanisms and potential therapeutic target. Intens Care Med. 2020; 46:586-90. 
[14] Mycroft-West C, Su D, Elli S, Guimond S, Miller G, Turnbull J, et al. The 2019 Coronavirus (SARS-CoV-2) Surface Protein (Spike) S1 Receptor Binding Domain Undergoes Conformational Change Upon Heparin Binding. Cold Spring Harbor L. bioRxiv [Preprint]. 2020. doi: https://doi.org/10.1101/2020.02.29.971093

[15] Kalra RS, Kandimalla R. Engaging the spikes: heparan sulfate facilitates SARS-CoV-2 spike protein binding to ACE2 and potentiates viral infection. Signal Transduct Target Ther. 2021;6(1):39.

[16] Tiwari V, Tandon R, Sankaranarayanan NV, Beer JC, Kohlmeir EK, Swanson-Mungerson M, Desai UR. Preferential recognition and antagonism of SARS-CoV-2 spike glycoprotein binding to 3- $O$-sulfated heparan sulfate. bioRxiv [Preprint]. 2020 Oct 8:2020.10.08.331751. 